\title{
Assessment of tumor size as a useful marker for the surgical staging of endometrial cancer
}

\author{
ROBERTO BERRETTA ${ }^{1}$, TITO SILVIO PATRELLI ${ }^{1}$, COSTANZA MIGLIAVACCA ${ }^{1}$, \\ MARTINO ROLLA ${ }^{1}$, LAURA FRANCHI ${ }^{1}$, MICHELA MONICA $^{1}$, \\ ALBERTO BACCHI MODENA $^{1}$ and SALVATORE GIZZO ${ }^{2}$ \\ ${ }^{1}$ Department of Surgical Sciences, University of Parma, Parma; \\ ${ }^{2}$ Department of Women and Child Health, University of Padua, Padua, Italy
}

Received January 3, 2014; Accepted February 24, 2014

DOI: $10.3892 /$ or.2014.3108

\begin{abstract}
Accumulating evidence suggests that the estimation of tumor size may improve endometrial cancer treatment. We conducted an observational study aimed at elucidating the association between tumor size and other universally accepted prognostic factors in order to identify suitable preoperative parameters which can guide surgery in a subgroup of early corpus endometrial cancer. We found that when tumor size increased, both stage and grading were significantly increased. Tumor size was correlated with CA 125 serum values, node metastasis and peritoneal cytology status. Patients who have grade 1 or 2 endometrioid corpus cancer, myometrial invasion $<50 \%$ and $\leq 3 \mathrm{~cm}$ largest tumor diameter can only be treated with hysterectomy. The tumor largest diameter should be evaluated as a preoperative parameter that indicates patients who do not require lymphadenectomy.
\end{abstract}

\section{Introduction}

Endometrial cancer is the most common malignancy of the female genital tract in developed countries, with an incidence rate of 12,9/100.000 women/year and a mortality rate of $2,4 / 100.000$ (1). More than 88,000 new cases a year are reported in the European Union (2). The incidence has considerably increased during the last three decades and it is currently the fourth most frequent cancer among women, exceeded only by cancer of the breast, lung, colon and rectum (3), representing the seventh cause of cancer-related mortality among women in Western Europe (2). In industrialized countries, most cases of adenocarcinoma of the endometrium are diagnosed at an early stage with an overall survival rate of $\sim 90 \%$ (2).

Correspondence to: Dr Salvatore Gizzo, Department of Women and Child Health, Gynecologic and Obstetric Unit, University of Padua, Via Giustiniani 3, I-35128 Padua, Italy

E-mail: ginecologia_padova@libero.it

Key words: endometrial cancer, tumor volume size, pelvic lymphadenectomy, tumor markers, prognostic factors
The cancer spread pathways are: direct expansion, free transtubal implantation, blood and lymphatic invasion. The lymphatic spread is the most frequent pathway occurring three times more than the blood spread, and it allows malignant cells to reach the parametrium, vagina, ovaries and retroperitoneal, pelvic and para-aortic lymph nodes (4). In general, this type of cancer primarily involves the pelvic lymph nodes: external iliac, internal iliac, common iliac (medial and lateral) and obturator (external and internal in relation with the obturator nerve). Other patterns of lymphatic spread are the presacral, inguinal and lombo-aortic lymph nodes (i.e. para-caval, precaval, retro-caval, right lateral aortic). In relation to grading G1-G3, myometrial invasion and International Federation of Gynecology and Obstetrics (FIGO) staging, pelvic positive lymph nodes are reported in $8-15 \%$ of patients in clinical stage I, $30 \%$ of patients in clinical stage II and in $45 \%$ of patients in clinical stage III (5).

Among the pelvic lymph nodes, the external iliac nodes are the most frequently affected by metastasis both in tumors limited to the uterine corpus and to the cervix. An earlier spread to the common iliac lymph nodes is reported in the latter one. Para-aortic lymph nodes (1-6\% of cases) are rarely involved as the primary station, indeed they are mainly associated with metastatic pelvic lymph nodes (6). As demonstrated by several authors, risk factors for the recurrence of endometrial carcinoma can be divided into uterine and extrauterine factors (4). Uterine factors include histologic type, grade (7), depth of myometrial invasion (8), cervical involvement (4), vascular invasion $(9,10)$, presence of atypical endometrial hyperplasia (11), hormone receptor status and DNA ploidy (12).

Extrauterine factors include adnexal involvement, intraperitoneal metastasis, positive peritoneal cytology $(13,14)$ and pelvic and para-aortic lymph node metastasis $(7,15)$. Patients with no evidence of extrauterine disease, no cervical involvement and no evidence of vascular invasion are at low overall risk of recurrence. Grade and depth of invasion are important prognostic factors for these patients. Women with evidence of extrauterine disease, cervical involvement or vascular invasion constitute a high risk group. If one of these three factors is positive, the frequency of recurrence is $20 \%$, increasing to $43 \%$ for two positive factors and $63 \%$ for three factors. 
Furthermore, some clinical factors assume prognostic value; these include, BMI, age, race, socioeconomic status and previous use of tamoxifen as hormonal treatment after breast cancer (16). It has also been demonstrated that younger women have a more favorable prognosis due to a significantly higher proportion of early stage disease and less myometrial invasion (4).

In relation to the risk of recurrence in stage $\mathrm{I}$, this can be divided into three categories: low risk (stage IA G1 G2 with endometrioid type), intermediate risk (stage IA G3 with endometrioid type, stage IB G1 and G2 with endometrioid type), high risk (stage IB G3 with endometrioid type, all stages with non-endometrioid type) (2). The standard approach for endometrial cancer is surgery which consists in total hysterectomy, bilateral salpingo-oophorectomy, colpectomy of the superior third and peritoneal washing (17).

FIGO recommends the evaluation of pelvic and para-aortic lymph nodes in every case, but a few studies have challenged its utility. The ASTEC study in particular examined lymphadenectomy in $>1,400$ patients from 85 centres in 4 different states and showed that lymphadenectomy does not provide improvement in low risk cancer (stage IA, G1-G2) (18). Lymphadenectomy, by contrast, is necessary in intermediatehigh risk cancer to guide surgical staging and therapeutic choices.

The approach for stage II endometrial cancer consists in radical hysterectomy with bilateral salpingo-oophorectomy and systematic pelvic lymphadenectomy, with or without paraaortic lymphadenectomy (17). Maximal surgical debulking is imperative in stage III or IV cancer.

The aim of the present study was to assess tumor size in patients undergoing surgical staging for endometrial cancer in order to verify a correlation between this and the most important prognostic factors, and to identify a novel helpful element in planning surgical approach.

\section{Patients and methods}

One hundred and forty-seven patients with endometrial cancer treated at the Gynecologic-Obstetric Clinic of the University of Parma from August 2000 to January 2012 participated in the present study. All the enrolled patients were properly informed regarding the collection of data and they provided a written consent form to the use of data respecting their privacy (Italian law 675/96).

All patients underwent a surgical approach according to current international guidelines; laparotomic hysterectomy with bilateral salpingo-oophorectomy and systematic pelvic lymphadenectomy in 129 (87.8\%) patients and laparoscopic hysterectomy with bilateral salpingo-oophorectomy and systematic pelvic lymphadenectomy in $18(12.2 \%)$. We studied both patients undergoing follow-up and patients with no follow-up.

In the present retrospective study, the following inclusion criteria were used: histological diagnosis of endometrial endometrioid, primitive cancer, no important comorbidity, treatment with surgical lymphadenectomy, measurement of the largest dimension of the tumor by the pathologist. We collected data about cancer prognostic factors: FIGO stage, grading, peritoneal washing, positive lymph nodes. This
Table I. Detailed data regarding surgical and pathological features of patients.

Total, n (\%)

\begin{tabular}{lc}
\hline Patients & $147(100)$ \\
Stage & $147(100)$ \\
IA & $67(45.6)$ \\
IB & $49(33.3)$ \\
II & $6(4.1)$ \\
IIIA & $9(6.1)$ \\
IIIB & $0(0.0)$ \\
IIIC & $16(10.9)$ \\
IVA & $0(0.0)$ \\
IVB & $0(0.0)$ \\
Grading & $147(100)$ \\
1 & $64(43.5)$ \\
2 & $66(44.9)$ \\
3 & $17(11.6)$ \\
Peritoneal cytologic results & $122(83)$ \\
Positive & $4(3.3)$ \\
Negative & $118(96.7)$ \\
Lymphadenectomy & $147(100)$ \\
Positive & $16(10.9)$ \\
Negative & $131(89.1)$ \\
\hline
\end{tabular}

information is kept in the Gynecologic ward archive and in the central archive of our hospital.

We also gathered data regarding tumor markers CA 125 reported in the patients' medical reports. We revised the patients' staging in order to conform them to the 2009 FIGO staging review. All tumors were measured by the expert pathologist and were reported in the original pathology reports. According to other authors and histological reports available, we considered the largest diameter.

We studied the state of the lymph nodes considering positive or negative nodes independently of their number or percentage and we correlated primary diameters with other prognostic factors such as stage, grading, peritoneal cytologic results, lymph node metastasis tumor markers.

Statistical analysis was performed by SPSS (Chicago, IL, USA) software for Windows version 19, using parametric and non-parametric tests where appropriate. We performed the Kolmogorov-Smirnov test for the normality of distribution. Continuous data were tested with the t-test, and categorical variables were tested with the $\chi^{2}$ test or Fisher's exact test where appropriate. The results obtained from the data collection are expressed in absolute number and percentage for discrete variables, in means \pm standard deviation for continuous variables. Statistical significance of differences was defined as $\mathrm{p}<0.05$.

\section{Results}

The distribution of the FIGO stage of the study group showed 67 patients $(45.6 \%)$ in stage IA, $49(33.3 \%)$ in stage IB, 
Table II. Correlation between primary tumor diameter and stage, grading, peritoneal cytology and lymph node status.

\begin{tabular}{lcc}
\hline Variables & $\begin{array}{c}\text { Tumor diameter } \\
\text { mean }( \pm \mathrm{SD})\end{array}$ & P-value \\
\hline Stage & & \\
IA & $2.9(2.3)$ & 0.01 \\
IB & $4.4(2.5)$ & 0.01 \\
II & $9.3(3.9)$ & 0.01 \\
IIIA & $5.4(3)$ & 0.01 \\
IIIC & $6.3(3.1)$ & 0.01 \\
Grading & & \\
1 & $3.3(2.6)$ & 0.01 \\
2 & $4.8(3.2)$ & 0.01 \\
3 & $5.8(2.5)$ & 0.01 \\
Peritoneal cytologic results & & \\
Positive & $7.5(2.6)$ & $<0.05$ \\
Negative & $4.1(2.8)$ & $<0.05$ \\
Lymph node status & & 0.01 \\
Positive & $6.3(3.1)$ & 0.01 \\
Negative & $4(2.8)$ & \\
\hline
\end{tabular}

$6(4.1 \%)$ in stage II, $9(6.1 \%)$ in stage IIIA and $16(10.9 \%)$ in stage IIIC. There were no patients in stage IV. The grading distribution indicated 64 cases (43.5\%) G1, 66 (44.9\%) G2 and $17(11.6 \%)$ G3. Peritoneal washings for cytologic assessment were available from 122 patients. Peritoneal cytologic results were negative in $118(96.7 \%)$ patients and positive in $4(3.3 \%)$.

All patients underwent pelvic lymphadenectomy, the median of the lymph nodes removed was 16 ; in $131(89.1 \%)$ cases nodes were negative, in $16(10.9 \%)$ cases they were positive (Table I). CEA was available for 25 patients, CA 125 for 83, CA 19.9 for 58 and CA 15.3 for 57.

We correlated tumor size with all these variables (Table II). The mean tumor largest diameter was 4.26 (range 0.4-13 cm). Tumor size was significantly related to FIGO stage $(\mathrm{p}<0.01)$ (Fig. 1). The average tumor dimension in stage IA was $2.9 \mathrm{~cm}( \pm 2.3$; median, $2.2 \mathrm{~cm}), 4.4 \mathrm{~cm}( \pm 2.5)$ in stage IB (median, $3.5 \mathrm{~cm}), 9.6 \mathrm{~cm}( \pm 3.9)$ in stage II (median, $10 \mathrm{~cm}$ ), $5.4 \mathrm{~cm}( \pm 3)$ in stage IIIA (median, $6 \mathrm{~cm})$, and $6.3 \mathrm{~cm}( \pm 3.1)$ in stage IIIC (median, $6.5 \mathrm{~cm}$ ).

Histological grading also correlated with the tumor largest diameter $(\mathrm{p}<0.01)$. When the tumor grade was 1 the mean size was $3.3 \mathrm{~cm}( \pm 2.6$; median, $2.5 \mathrm{~cm})$; when it was 2 , the average diameter was $4.8 \mathrm{~cm}( \pm 3.2$; median, $3.2 \mathrm{~cm})$, and when the tumors were undifferentiated, grade 3 , the mean dimension was $5.8 \mathrm{~cm}( \pm 2.5$; median, $5.5 \mathrm{~cm})$ (Fig. 2).

The most statistically significant difference was between the mean tumor size of grade 1 and 2 cancer and between the mean tumor size of grade 1 and 3 tumor $(\mathrm{p}=0.012)$ as calculated by the post-hoc test. There were no notable differences between other comparisons.

A marked correlation existed between tumor largest diameter and nodal metastases (Fig. 3). The mean tumor size was $4 \mathrm{~cm}( \pm 2.8)$ and the median was $3 \mathrm{~cm}$ when lymph nodes

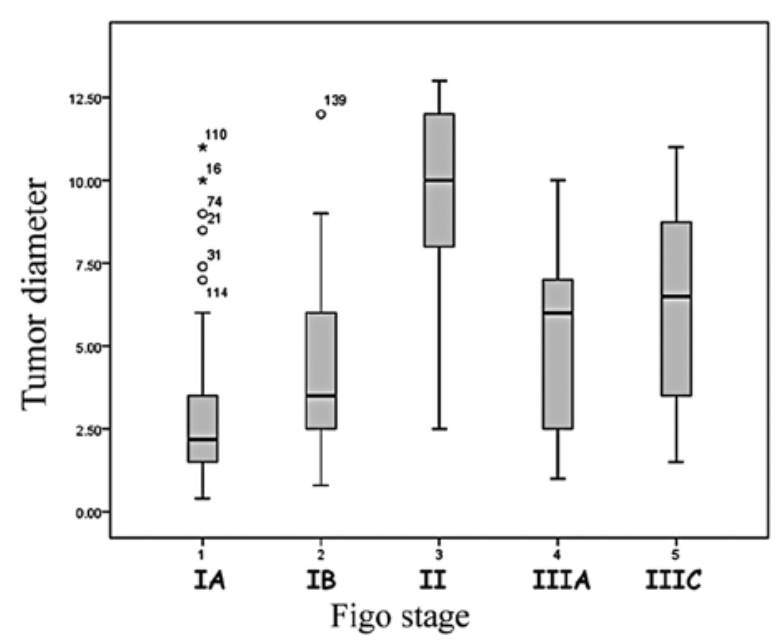

Figure 1. Correlation between tumor mean size and FIGO stage.

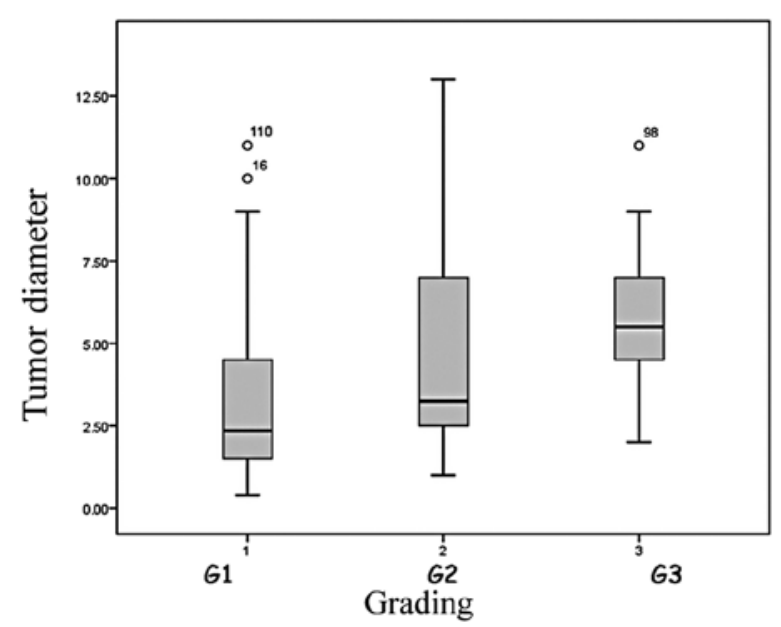

Figure 2. Correlation between tumor mean size and histological grade.

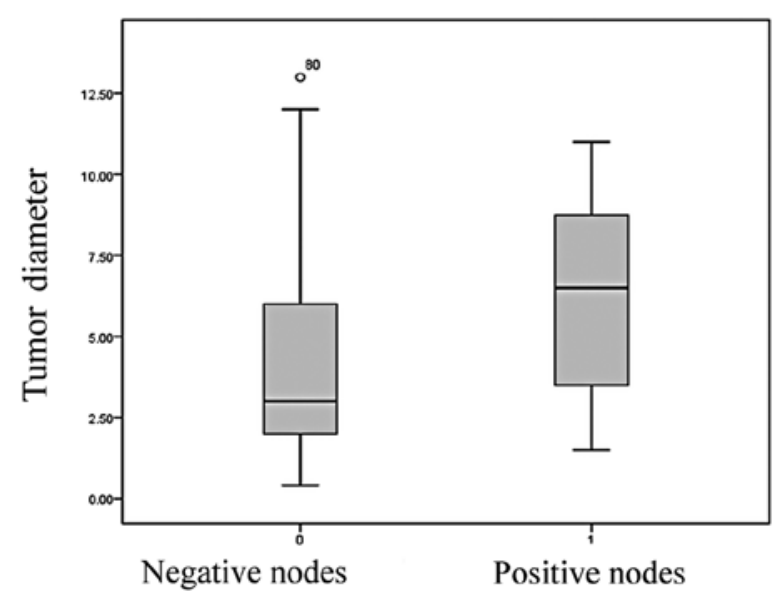

Figure 3. Correlation between tumor mean size and nodal metastasis.

resulted negative. The average dimension of tumor with nodal metastases was $6.3 \mathrm{~cm}( \pm 3.1)$ and the median was $6.5 \mathrm{~cm}$. The correlation coefficient here was $0.003(\mathrm{p}<0.01)$. 


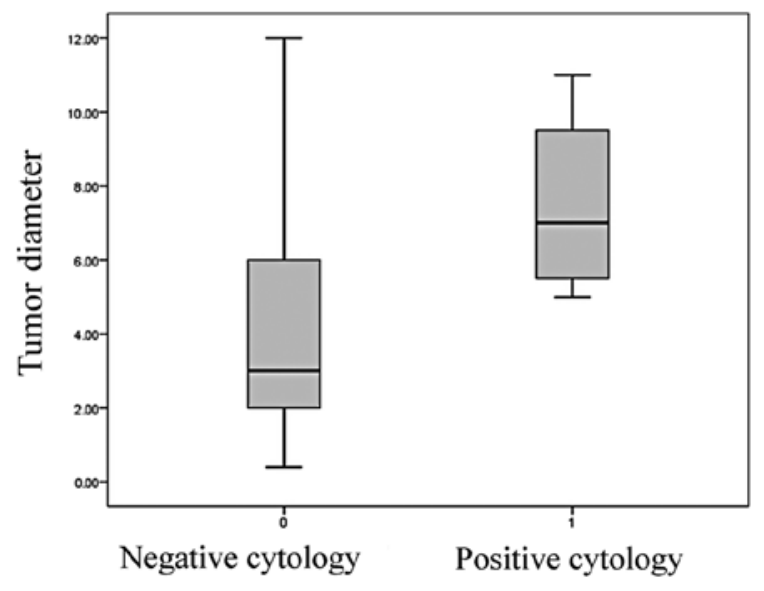

Figure 4. Correlation between tumor mean size and peritoneal cytology results.

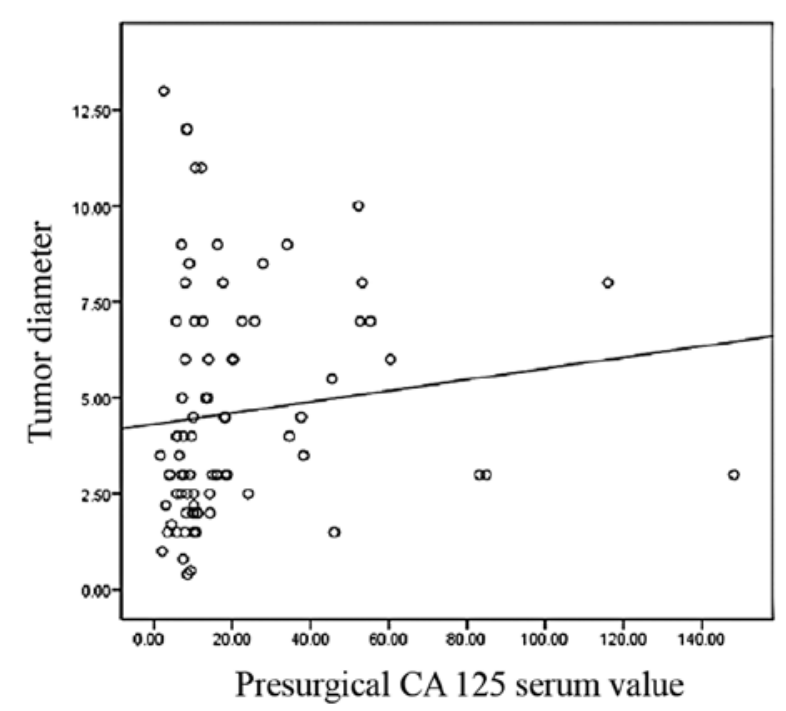

Figure 5. Correlation between tumor mean size and CA 125 serum value.

The t-test also demonstrated an association between peritoneal cytologic results and tumor size (Fig. 4). Types of cancers with negative peritoneal cytologic results were smaller $(4.1 \pm 2.8 \mathrm{~cm})$ than cancers with negative results $(7.5 \pm 2.6 \mathrm{~cm})$ $(\mathrm{p}=0.020)$. A correlation $(\mathrm{p}=0.009)$ existed between preoperative CA 125 values and tumor largest diameter (Fig. 5).

\section{Discussion}

Endometrial cancer, different from non-epithelial cancer of corpus uteri $(19,20)$, is a gynecological malignancy with an elevated prevalence and a low mortality rate in the initial stages, especially in developed countries. These data are a result of the greater number of diagnostic instruments, increased average life expectancy and diagnosis in older patients.

According to recent literature (2), patients can be divided into two groups based on the biological character of the malignancy. These two groups, high risk (undifferentiated cancer of every stage or deeply infiltrating cancer besides the stage) and low-risk (initially infiltrating cancer, G1-G2) undergo different approaches; complete intensive surgical staging in high-risk cancers while there is a less invasive approach in low-risk cancers.

Due to this, the scientific community has been interested in detecting new instruments to identify these two groups of patients before surgery. Grading is already a suitable indicator of the necessity of lymphadenectomy. Other parameters such as myometrial invasion or cervix involvement have been studied with ultrasound or magnetic resonance. It is generally agreed that in the assessment of the depth of myometrial invasion by endometrial carcinoma, TV-ultrasound has a high diagnostic accuracy that is equivalent to that of magnetic resonance imaging (MRI). MRI, in addition, can give more information about cervical or parametrial involvement $(21,22)$. Tumor sizes are easily assessable by the use of current diagnostic techniques such as hysteroscopy and ultrasound imaging.

In this retrospective study, analyzing the cancer macroscopic dimensions, we found that tumor stage and grading increase as tumor size increases. Nodal metastases and positive peritoneal cytologic results risk increase too, therefore the risk of extrauterine disease increases in the largest lesions. Indeed, an association between tumor largest diameter and FIGO stage was demonstrated. This correlation was not perfectly linear since the mean size of the tumor in stage II $(9.6 \pm 3.9 \mathrm{~cm})$ was higher than that of the tumor in stage IIIA $(5.4 \pm 3 \mathrm{~cm})$. We suppose that this data has been distorted by the number of patients in the present study; the patients in stage II were significantly fewer than patients in other stages, and among the six patients in stage II, one of them developed a large cancer.

An important difference between the size of cancer in stage IA $(2.9 \pm 2.3 \mathrm{~cm})$ and IB $(4.4 \pm 2.5 \mathrm{~cm})$ was reported in the present study. The difference between these two stages consists in the invasion of more or less than $50 \%$ of the myometrium; therefore, the present study, according to previous literature $(23,24)$, demonstrates that the probability of myometrial invasion increases with the tumor size.

According to our data, grading and tumor dimension are related. In particular, we noted a maximum correlation between well differentiated and highly undifferentiated cancer in relation to tumor size.

In contrast to Mariani et al (24), we correlated peritoneal cytologic results with tumor size. In our study, $88 \%$ of patients underwent peritoneal washing and only $3.3 \%$ had positive peritoneal cytologic results. The present study shall be continued, in order to verify the underlying significance of this correlation.

There are no studies that relate tumor size with tumor markers. The present study showed an association between tumor diameter and CA 125. CA 125 correlates with FIGO stage which in turn is associated with tumor size in this study (25). A correlation between CA 125 and tumor size derives from these data and further confirms our findings. Different from CA 125, that often results increased in a non-oncologic disease such as endometriosis (26), HE4 was recently proposed as a diagnostic and prognostic marker in patients affected by endometrial cancer. Despite HE4 showing better sensitivity and specificity (albeit in early stages) than CA 125, it requires further validation and estimation concerning the best cut-off value, particularly in the case of coexisting chronic disease as renal impairment (27). 
Most of the literature focuses on the association between tumor size and nodal metastases. In 1960, Gusberg et al demonstrated a poorer prognosis when the tumor was $>10 \mathrm{~cm}$ (28). In 1979, Johnsson determined an increase of extrauterine disease frequency in tumors larger than a third of the uterine cavity (29). Shink et al (30) and Lurain et al (31) showed a decrease in the risk of lymph node metastases and an increase of survival in tumors $<2 \mathrm{~cm}$.

The present study, according to these data, demonstrated an important association between tumor size and the risk of lymph node metastases. Among our 147 patients, $89.1 \%$ had no nodal metastases; the mean tumor size in this group of patients was $4.1 \mathrm{~cm}( \pm 2.8)$ and the median was $3 \mathrm{~cm}$. This value comes significantly close to the cut-off of $2 \mathrm{~cm}$ defined first by Shink et al $(23,30)$, and then demonstrated in other studies $(24,31,32)$.

Considering these data and particularly the demonstrated association between tumor size and myometrial invasion, grading, nodal metastases, the present study is in accordance with Mariani et al (24) and Milam et al (32) and proposes the consideration of endometrioid adenocarcinoma of the endometrium, grade 1 or 2 , with myometrial invasion $<50 \%$ and tumor largest diameter $\leq 3 \mathrm{~cm}$ as low-risk of nodal metastases. In accordance with these authors, we maintain that it is reasonably safe to treat with lymphadenectomy patients who are, according to these criteria, at high-risk of metastases. According to Mariani et al (24), patients with positive peritoneal cytologic results should undergo lymphadenectomy, but the 2009 FIGO staging review does not include peritoneal cytologic results among staging parameters, therefore we consider it the less important parameter in the identification of patient risk.

According to the recent literature, we concluded that tumor size is correlated with stage and grade, which are important prognostic factors to determine therapeutic approach. We also found a correlation between tumor size and nodal metastasis, positive peritoneal cytology and CA 125 values. Patients who have grade 1 or 2 endometrioid corpus cancer, myometrial invasion $<50 \%$ and largest tumor diameter $\leq 3 \mathrm{~cm}$ can be treated only with hysterectomy. Tumor largest diameter should be evaluated as a preoperative parameter that indicates patients who do not require lymphadenectomy.

The preoperative assessment of tumor size using imaging techniques such as hysteroscopy (33), ultrasonography or magnetic resonance, should be included in the diagnosis of patients with suspected endometrial cancer in order to guide the surgeon to determine the most appropriate surgical strategy for each patient.

\section{Acknowledgements}

The authors acknowledge all the staff of the OB/GYN Unit of Pharma University for their help in collecting the data.

\section{References}

1. Jemal A, Bray F, Center MM, Ferlay J, Ward E and Forman D Global cancer statistics. CA Cancer J Clin 61: 69-90, 2011.

2. Colombo N,Preti E, Landoni F, Carinelli S, Colombo A, Marini C, Sessa C; ESMO Guidelines Working Group: Endometrial cancer: ESMO Clinical Practice Guidelines for diagnosis, treatment and follow-up. Ann Oncol 22 (Suppl 6): 35-39, 2011.
3. Amant F, Moerman P, Neven P, Timmerman D, Van Limbergen E and Vergote I: Endometrial cancer. Lancet 366: 491-505, 2005.

4. Patrelli TS, Berretta R, Rolla M, Vandi F, Capobianco G, Gramellini D, Bacchi Modena A and Nardelli GB: Pelvic lymphadenectomy in endometrial cancer: our current experience. Eur $\mathrm{J}$ Gynaecol Oncol 30: 536-538, 2009.

5. Zhang C, Wang C and Feng W: Clinicopathological risk factors for pelvic lymph node metastasis in clinical early-stage endometrioid endometrial adenocarcinoma. Int J Gynecol Cancer 22: 1373-1377, 2012.

6. Mariani A, Webb MJ, Keeney GL and Podratz KC: Routes of lymphatic spread: a study of 112 consecutive patients with endometrial cancer. Gynecol Oncol 81: 100-104, 2001.

7. Morrow CP, Bundy BN, Kurman RJ, Creasman WT, Heller P, Homesley HD and Graham JE: Relationship between surgicalpathological risk factors and outcome in clinical stage I and II carcinoma of the endometrium: a Gynecologic Oncology Group study. Gynecol Oncol 40: 55-65, 1991.

8. Berretta R, Merisio C, Piantelli G, Rolla M, Giordano G, Melpignano $M$ and Nardelli GB: Preoperative transvaginal ultrasonography and intraoperative gross examination for assessing myometrial invasion by endometrial cancer. J Ultrasound Med 27: 349-355, 2008

9. Al Kushi A, Lim P, Aquino-Parsons C and Gilks CB: Markers of proliferative activity are predictors of patient outcome for low-grade endometrioid adenocarcinoma but not papillary serous carcinoma of endometrium. Mod Pathol 15: 365-371, 2002.

10. Nofech-Mozes S, Ackerman I, Ghorab Z, Ismiil N, Thomas G, Covens A and Khalifa MA: Lymphovascular invasion is a significant predictor for distant recurrence in patients with earlystage endometrial endometrioid adenocarcinoma. Am J Clin Pathol 129: 912-917, 2008.

11. Merisio C, Berretta R, De Ioris A, Pultrone DC, Rolla M, Giordano G, Tateo S and Melpignano M: Endometrial cancer in patients with preoperative diagnosis of atypical endometrial hyperplasia. Eur J Obstet Gynecol Reprod Biol 122: 107-111, 2005.

12. Di Cristofano A and Ellenson LH: Endometrial carcinoma. Annu Rev Pathol 2: 57-85, 2007.

13. Gu M, Shi W, Barakat RR, Thaler HT and Saigo PE: Peritoneal washings in endometrial carcinoma. A study of 298 patients with histopathologic correlation. Acta Cytol 44: 783-789, 2000.

14. Turner DA, Gershenson DM, Atkinson N, Sneige N and Wharton AT: The prognostic significance of peritoneal cytology for stage I endometrial cancer. Obstet Gynecol 74: 775-780, 1989.

15. Hanson MB, van Nagell JR Jr, Powell DE, Donaldson ES, Gallion H, Merhige M and Pavlik EJ: The prognostic significance of lymph-vascular space invasion in stage I endometrial cancer. Cancer 55: 1753-1757, 1985.

16. Gizzo S, Di Gangi S, Bertocco A, Noventa M, Fagherazzi S, Ancona E, Saccardi C, Patrelli TS, D'Antona D and Nardelli GB: Levonorgestrel intrauterine system in adjuvant tamoxifen treatment: balance of breast risks and endometrial benefits systematic review of literature. Reprod Sci: Sep 23, 2013 (Epub ahead of print).

17. Wright JD, Barrena Medel NI, Sehouli J, Fujiwara K and Herzog TJ: Contemporary management of endometrial cancer. Lancet 379: 1352-1360, 2012.

18. ASTEC study group, Kitchener H, Swart AM, Qian Q, Amos C and Parmar MK: Efficacy of systematic pelvic lymphadenectomy in endometrial cancer (MRC ASTEC trial): a randomised study. Lancet 373: 125-136, 2009.

19. Patrelli TS, Silini EM, Gizzo S, Berretta R, Franchi L, Thai E, Lukanovic A, Nardelli GB and Modena AB: Extragenital Müllerian adenosarcoma with pouch of Douglas location. BMC Cancer 11: 171, 2011.

20. Patrelli TS, Gizzo S, Di Gangi S, Guidi G, Rondinelli M and Nardelli GB: Cervical Mullerian adenosarcoma with heterologous sarcomatous overgrowth: a fourth case and review of literature. BMC Cancer 11: 236, 2011.

21. Savelli L, Testa AC, Mabrouk M, Zannoni L, Ludovisi M, Seracchioli R, Scambia G and De Iaco P: A prospective blinded comparison of the accuracy of transvaginal sonography and frozen section in the assessment of myometrial invasion in endometrial cancer. Gynecol Oncol 124: 549-552, 2012.

22. Savelli L, Ceccarini M, Ludovisi M, Fruscella E, De Iaco PA, Salizzoni E, Mabrouk M, Manfredi R, Testa AC and Ferrandina G: Preoperative local staging of endometrial cancer: transvaginal sonography vs. magnetic resonance imaging. Ultrasound Obstet Gynecol 31: 560-566, 2008. 
23. Schink JC, Rademaker AW, Miller DS and Lurain JR: Tumor size in endometrial cancer. Cancer 67: 2791-2794, 1991.

24. Mariani A, Webb MJ, Keeney GL, Haddock MG, Calori G and Podratz KC: Low-risk corpus cancer: is lymphadenectomy or radiotherapy necessary? Am J Obstet Gynecol 182: 1506-1519, 2000.

25. Sorosky JI: Endometrial cancer. Obstet Gynecol 111: 436-447, 2008.

26. Patrelli TS, Berretta R, Gizzo S, Pezzuto A, Franchi L, Lukanovic A, Nardelli GB and Modena AB: CA 125 serum values in surgically treated endometriosis patients and its relationships with anatomic sites of endometriosis and pregnancy rate. Fertil Steril 95: 393-396, 2011.

27. Gizzo S, Ancona E, Saccardi C, D'Antona D, Nardelli GB and Plebani M: Could kidney glomerular filtration impairment represent the "Achilles heel" of HE4 serum marker? A possible further implication. Clin Chem Lab Med 52: e45-e46, 2014.

28. Gusberg SB, Jones HC Jr and Tovell HM: Selection of treatment for corpus cancer. Am J Obstet Gynecol 80: 374-380, 1960.
29. Johnsson JE: Recurrences and metastases in carcinoma of the uterine body correlated to the size and localization of the primary tumor. Acta Obstet Gynecol Scand 58: 405-408, 1979.

30. Schink JC, Lurain JR, Wallemark CB and Chmiel JS: Tumor size in endometrial cancer: a prognostic factor for lymph node metastasis. Obstet Gynecol 70: 216-219, 1987.

31. Lurain JR, Rice BL, Rademaker AW, Poggensee LE, Schink JC and Miller DS: Prognostic factors associated with recurrence in clinical stage I adenocarcinoma of the endometrium. Obstet Gynecol 78: 63-69, 1991.

32. Milam MR, Java J, Walker JL, Metzinger DS, Parker LP, Coleman RL; Gynecologic Oncology Group: Nodal metastasis risk in endometrioid endometrial cancer. Obstet Gynecol 119: 286-292, 2012.

33. Saccardi C, Gizzo S, Patrelli TS, Ancona E, Anis O, Di Gangi S, Vacilotto A, D'Antona D and Nardelli GB: Endometrial surveillance in tamoxifen users: role, timing and accuracy of hysteroscopic investigation: observational longitudinal cohort study. Endocr Relat Cancer 20: 455-462, 2013. 